\title{
Intracolonial genetic variation affects reproductive skew and colony productivity during colony foundation in a parthenogenetic termite
}

\author{
Satoshi Miyazaki ${ }^{1,2}$, Miho Yoshimura ${ }^{1}$, Ryota Saiki ${ }^{1}$, Yoshinobu Hayashi ${ }^{3}$, Osamu Kitade ${ }^{4}$, Kazuki Tsuji ${ }^{5}$
} and Kiyoto Maekawa ${ }^{1 *}$

\begin{abstract}
Background: In insect societies, intracolonial genetic variation is predicted to affect both colony efficiency and reproductive skew. However, because the effects of genetic variation on these two colony characteristics have been tested independently, it remains unclear whether they are affected by genetic variation independently or in a related manner. Here we test the effect of genetic variation on colony efficiency and reproductive skew in a rhinotermitid termite, Reticulitermes speratus, a species in which female-female pairs can facultatively found colonies. We established colonies using two types of female-female pairs: colonies founded by sisters (i.e., sister-pair colonies) and those founded by females from different colonies (i.e., unrelated-pair colonies). Colony growth and reproductive skew were then compared between the two types of incipient colonies.
\end{abstract}

Results: At 15 months after colony foundation, unrelated-pair colonies were larger than sister-pair colonies, although the caste ratio between workers and nymphs, which were alternatively differentiated from young larvae, did not differ significantly. Microsatellite DNA analyses of both founders and their parthenogenetically produced offspring indicated that, in both sister-pair and unrelated-pair colonies, there was no significant skew in the production of eggs, larvae, workers and soldiers. Nymph production, however, was significantly more skewed in the sister-pair colonies than in unrelated-pair colonies. Because nymphs can develop into winged adults (alates) or nymphoid reproductives, they have a higher chance of direct reproduction than workers in this species.

Conclusions: Our results support the idea that higher genetic variation among colony members could provide an increase in colony productivity, as shown in hymenopteran social insects. Moreover, this study suggests that low genetic variation (high relatedness) between founding females increases reproductive skew via one female preferentially channeling her relatives along the reproductive track. This study thus demonstrated that, in social insects, intracolonial genetic variation can simultaneously affect both colony efficiency and reproductive skew.

Keywords: Intracolonial genetic variation, Colony efficiency, Reproductive skew, Eusociality, Parthenogenesis, Caste differentiation, Nymph, Reticulitermes speratus

\footnotetext{
* Correspondence: kmaekawa@sci.u-toyama.ac.jp

${ }^{1}$ Graduate School of Science and Engineering, University of Toyama,

930-8555 Gofuku, Toyama, Japan

Full list of author information is available at the end of the article
} 


\section{Background}

Recent evolutionary theories predict that genetic variation among group members can affect characteristics of social insect colonies in two ways. First, an increase in genetic variation among workers can enhance colony efficiency by facilitating division of labor [1] and increased disease resistance [2,3]. Second, increased genetic variation among colony members can affect intracolonial conflicts, often by intensifying them [4]. Although there is empirical evidence supporting the above views, to our knowledge, no study has tested the two effects simultaneously. Thus, it remains unknown whether genetic variation has a direct impact on both characteristics. A study testing these two effects would provide us an opportunity to understand how intracolonial genetic variation affects evolution and maintenance of colony characteristics.

We focused on colony founding in the rhinotermitid termite Reticulitermes speratus. In this species, as in other termites, newly emerged winged adults (alates) generally form a male-female pair, which founds a colony together and reproduces sexually [5]. However, Matsuura and Nishida [6] reported that occasionally when two female alates failed to find males, they could co-found a colony and reproduce parthenogenetically. This homosexual cooperation benefits both females, because their survival probability is markedly enhanced when compared to females singly founding a colony $[7,8]$. Given the limited dispersal (flight) ability of alate termites in this species, relatedness of such co-foundress naturally varies, depending on the distribution of colonies in a population. This termite is therefore suitable for testing the two possible predicted effects of intracolonial genetic variation.

Reproductive skew, the degree of uneven partitioning of reproductive output among group members, is a colony characteristic that can reflect intracolonial conflicts. Transactional models of reproductive skew, in which group members yield reproductive shares to each other in return for cooperation, predict that reproductive skew becomes larger as relatedness increases [9]. Empirical tests of this and other predictions of reproductive skew models are often problematic $[4,10]$, however, because predictions are affected by assumptions and by several parameters other than relatedness (i.e., group size, fighting ability, ecological effect on independent reproduction, etc., [11-13]). One such parameter that affects the outcome is group size. Many reproductive skew models assume that a group consists of two individuals (cooperating) or one individual (not-cooperating). However, the group size of real animals is often more than two individuals and can vary, giving rise to a statistical problem in defining reproductive skew [14]. Colony founding by $R$. speratus is advantageous in testing reproductive skew models, because the group size is either two (pair founding) or one (individual or haplometrotic founding), thus meeting a fundamental assumption of basic reproductive skew models [15].

The genetic factors associated with determining caste trajectory into nymphs vs. workers have been identified in this species; parthenogenetically produced eggs are genetically predisposed to develop into nymphs. However, a percentage of eggs can develop into workers in response to environmental stimuli, e.g., the presence of reproductive individuals [16]. Nymphs can molt into secondary reproductives (nymphoids; Figure 1a), and nymphoids parthenogenetically produced by the primary queen become the main reproductive force in natural colonies of this species [17]. Thus, the production of nymphs can be an index of reproductive output in colonies. Based on the above evolutionary theories, we tested our prediction that a reproductive skew in nymph production is affected by genetic relatedness of co-foundresses, and that genetic variation among sterile workers positively affects colony efficiency.

To test whether intracolonial genetic variation affects colony efficiency and reproductive skew in $R$. speratus, we studied two types of incipient female-female colonies under laboratory conditions: colonies founded by closely related individuals (i.e., sister-pair colonies) and those founded by non-kin (i.e., unrelated-pair colonies). Colonies were censused and compared at 15 months after colony foundation, and the genotypes of individual colony members were determined using microsatellite DNA.

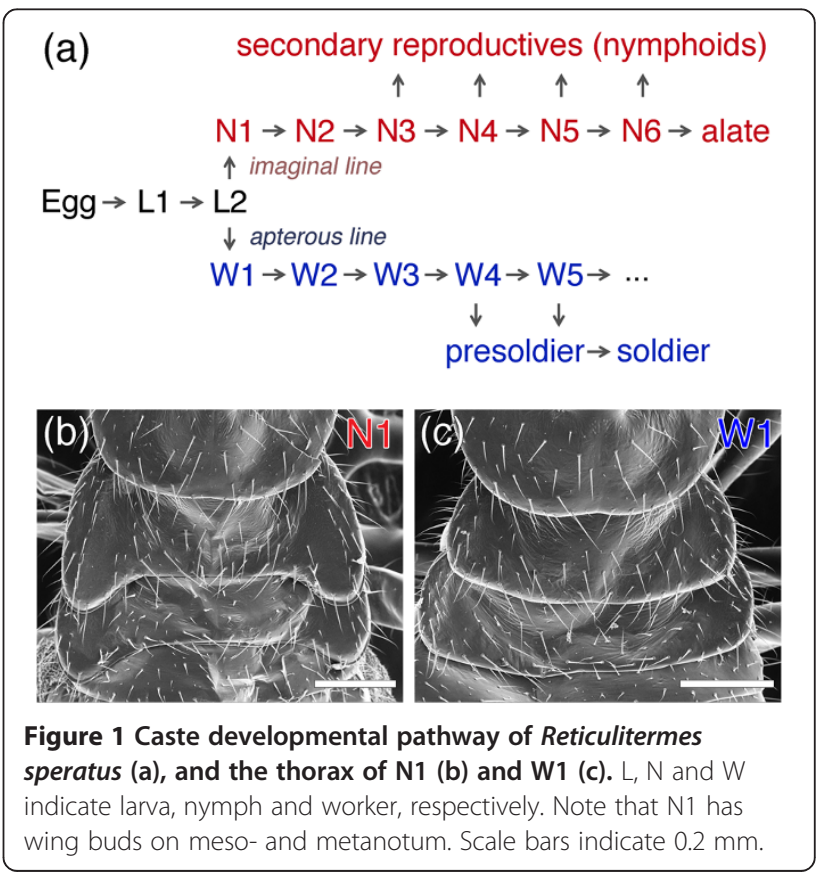




\section{Results}

Rapid colony development in unrelated-pair colonies At 15 months after colony foundation, 78.6 and $64.0 \%$ of founding females survived in 28 sister-pair colonies and 43 unrelated-pair colonies, respectively. The survival rates did not differ significantly between these two colony types (generalized linear mixed model (GLMM), Table 1). A GLMM analysis of the effects of colony characters including colony type (sister-pair or unrelated-pair) on colony size (the sum of numbers of queens, eggs, larvae, workers, soldiers, nymphs and nymphoids) revealed that the colony size was significantly affected by colony type, but neither the founders' survival rates nor the colony type $\times$ survival rate

Table 1 Effects of social characters on colony efficiency (the number of termites)

\begin{tabular}{|c|c|c|c|}
\hline Response variable & Explanatory variable & $Z$ value & $P$ value \\
\hline $\begin{array}{l}\text { Founders' survival } \\
\text { rate }\end{array}$ & colony type $(\mathrm{CT})$ & 1.835 & 0.067 \\
\hline \multirow[t]{3}{*}{ Colony size } & colony type $(C T)$ & -2.017 & $0.044^{*}$ \\
\hline & founders' survival rate (FSR) & -1.048 & 0.295 \\
\hline & interaction between CT \& FSR & 1.094 & 0.274 \\
\hline \multirow[t]{7}{*}{ Nymph/worker ratio } & colony type (CT) & -0.790 & 0.430 \\
\hline & founders' survival rate (FSR) & -0.533 & 0.594 \\
\hline & colony size (CS) & 0.306 & 0.759 \\
\hline & interaction between CT \& FSR & 1.149 & 0.251 \\
\hline & interaction between CT \& CS & 1.265 & 0.206 \\
\hline & interaction between FSR \& CS & 0.280 & 0.779 \\
\hline & $\begin{array}{l}\text { interaction between } C T \text {, } \\
\text { FSR \& CS }\end{array}$ & -1.433 & 0.152 \\
\hline \multirow[t]{7}{*}{ Number of soldiers } & colony type $(\mathrm{CT})$ & 0.405 & 0.685 \\
\hline & founders' survival rate (FSR) & 0.421 & 0.674 \\
\hline & colony size (CS) & -0.060 & 0.952 \\
\hline & interaction between CT \& FSR & -0.181 & 0.856 \\
\hline & interaction between CT \& CS & -0.872 & 0.383 \\
\hline & interaction between FSR \& CS & 0.010 & 0.992 \\
\hline & $\begin{array}{l}\text { interaction between } C T \text {, } \\
\text { FSR \& CS }\end{array}$ & 0.374 & 0.708 \\
\hline \multirow{7}{*}{$\begin{array}{l}\text { Number of } \\
\text { nymphoids }\end{array}$} & colony type (CT) & 0.165 & 0.869 \\
\hline & founders' survival rate (FSR) & -0.725 & 0.469 \\
\hline & colony size (CS) & -0.623 & 0.533 \\
\hline & interaction between CT \& FSR & -0.216 & 0.829 \\
\hline & interaction between CT \& CS & 0.119 & 0.905 \\
\hline & interaction between FSR \& CS & -0.029 & 0.977 \\
\hline & $\begin{array}{l}\text { interaction between CT, FSR \& } \\
\text { CS }\end{array}$ & -0.084 & 0.933 \\
\hline
\end{tabular}

Social characters include colony types (i.e., sister-pair and unrelated-pair colonies), founders' survival rate, colony size and their interactions.

"*" indicates $P<0.05$ in generalized linear mixed model (GLMM) analyses. interaction (Table 1). Specifically, colony size in unrelated-pair colonies was significantly larger than those in the sister-pair colonies (Figure 2a). Nymph/ worker ratio, however, was not affected by colony type (Figure 2b), colony size, number of surviving queens, nor any interactions among these factors (GLMM, Table 1), indicating that intracolonial genetic variation between founders did not affect the nymph-worker caste ratio at the colony level. The number of neither nymphoids nor soldiers differed between colony types (GLMM, Table 1).

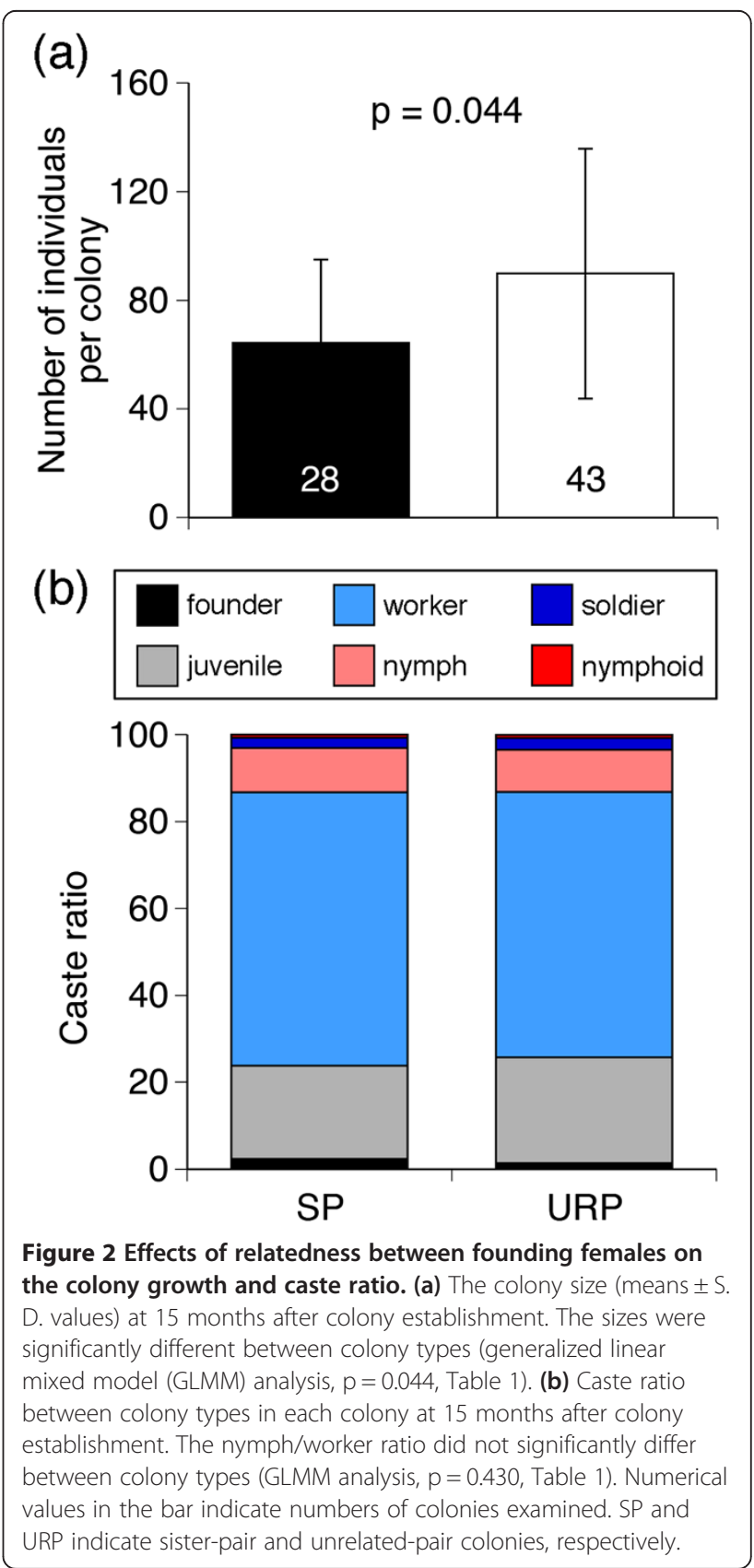




\section{Nymph production skewed toward one of the paired founding females in sister-pair colonies}

Genetic differences (shown as different microsatellite alleles) between the paired founding females were observed (or estimated from worker genotypes, in cases of colonies with only 1 female surviving; see Methods) in 13 sister-pair and 14 unrelated-pair colonies. In these colonies, microsatellite DNA analyses were performed to assign parentage to offspring, which included juveniles (eggs and larvae), workers, soldiers, nymphs and nymphoids (Additional file 1 and Additional file 2); the higher proportion $(\geqq 50 \%)$ between two founding females was then defined as the skew of offspring production. The skew of nymph production was significantly affected by colony type, both directly and indirectly, while the skew of juvenile-, worker-, soldier-, and offspringproduction were not (Table 2, GLMM). Colony size and survival rate of founding females did not significantly affect skews (Table 2, GLMM). These results indicate that nymph production was more skewed in sister-pair colonies than in unrelated-pair colonies (Figure 3, Additional file 3).

\section{Discussion}

In this study, we showed that genetic variation among colony members affected colony characteristics in $R$. speratus from two perspectives. First, in unrelated-pair colonies, colony growth was higher. Second, in sister-pair colonies, nymph production was skewed toward one of paired females. These results demonstrate that intracolonial genetic variation had the two distinct effects on colony characteristics: a positive effect on colony efficiency, and a negative effect on reproductive monopoly, i.e., reproductive skew.

\section{Colony growth increased in unrelated-pair colonies with higher genetic variation among workers}

In the honeybee Apis mellifera, intracolonial genetic variation promotes both task specialization and resistance to diseases, resulting in increases in colony productivity [1]. Similar relationships between genetic variation and colony productivity were observed in ants [18-20] and a wasp [21], taxa whose socialities evolved independently of the honeybee's [22,23]. Our results showed that, in R. speratus, colony size was larger in unrelated-pair colonies (i.e., with higher intracolonial genetic variation), than in sister-pair colonies (Figure 2a). Furthermore, in both sister-pair and unrelated-pair colonies, worker production was only slightly skewed toward one founding female (Figure 3), suggesting that there is an advantage to increasing genetic variation among workers. In a termite society, then, the increase in worker genetic variation promotes colony productivity, probably through effective division of labor and resistance to diseases, as in social hymenopterans.
Table 2 Effects of social characters on reproductive skew

\begin{tabular}{|c|c|c|c|}
\hline $\begin{array}{l}\text { Response } \\
\text { variable }\end{array}$ & Explanatory variable & $Z$ value & $P$ value \\
\hline \multirow{7}{*}{$\begin{array}{l}\text { Skew of offspring } \\
\text { production }\end{array}$} & colony type $(\mathrm{CT})$ & 1.952 & 0.051 \\
\hline & founders' survival rate (FSR) & 1.558 & 0.119 \\
\hline & colony size (CS) & 1.141 & 0.254 \\
\hline & interaction between CT \& FSR & -1.738 & 0.082 \\
\hline & interaction between CT \& CS & -1.585 & 0.113 \\
\hline & interaction between FSR \& CS & -1.534 & 0.125 \\
\hline & interaction between CT, FSR \& CS & 1.493 & 0.136 \\
\hline \multirow{7}{*}{$\begin{array}{l}\text { Skew of juvenile } \\
\text { production }\end{array}$} & colony type $(\mathrm{CT})$ & 1.482 & 0.138 \\
\hline & founders' survival rate (FSR) & 0.422 & 0.673 \\
\hline & colony size (CS) & 0.301 & 0.763 \\
\hline & interaction between CT \& FSR & -1.336 & 0.182 \\
\hline & interaction between CT \& CS & -1.365 & 0.172 \\
\hline & interaction between FSR \& CS & -0.342 & 0.733 \\
\hline & interaction between CT, FSR \& CS & 1.283 & 0.199 \\
\hline \multirow{7}{*}{$\begin{array}{l}\text { Skew of worker } \\
\text { production }\end{array}$} & colony type $(C T)$ & 1.232 & 0.218 \\
\hline & founders' survival rate (FSR) & 1.131 & 0.258 \\
\hline & colony size (CS) & 1.232 & 0.218 \\
\hline & interaction between CT \& FSR & -1.434 & 0.152 \\
\hline & interaction between CT \& CS & -1.315 & 0.189 \\
\hline & interaction between FSR \& CS & -1.581 & 0.114 \\
\hline & interaction between CT, FSR \& CS & 1.502 & 0.133 \\
\hline \multirow{7}{*}{$\begin{array}{l}\text { Skew of nymph } \\
\text { production }\end{array}$} & colony type $(\mathrm{CT})$ & 1.997 & $0.046^{*}$ \\
\hline & founders' survival rate (FSR) & -0.230 & 0.818 \\
\hline & colony size (CS) & 0.175 & 0.861 \\
\hline & interaction between CT \& FSR & -1.197 & 0.231 \\
\hline & interaction between CT \& CS & -2.664 & $0.008^{* *}$ \\
\hline & interaction between FSR \& CS & 0.049 & 0.961 \\
\hline & interaction between CT, FSR \& CS & 2.152 & $0.031^{*}$ \\
\hline \multirow{7}{*}{$\begin{array}{l}\text { Skew of soldier } \\
\text { production }\end{array}$} & colony type $(\mathrm{CT})$ & -0.001 & 0.999 \\
\hline & founders' survival rate (FSR) & 0.452 & 0.651 \\
\hline & colony size (CS) & 0.287 & 0.774 \\
\hline & interaction between CT \& FSR & 0.001 & 0.999 \\
\hline & interaction between CT \& CS & 0 & 1.000 \\
\hline & interaction between FSR \& CS & -0.211 & 0.833 \\
\hline & interaction between CT, FSR \& CS & 0 & 1.000 \\
\hline
\end{tabular}

For the explanatory values, see Table 1. " $* "$ and $" * * *$ indicates $\mathrm{P}<0.05$ and 0.01 in GLMM analyses, respectively. Skew of nymphoids could not be analyzed because of small numbers of colonies producing nymphoids ( 3 of 13 sister-pair colonies and 3 of 14 unrelated-pair ones, see Additional files 1, 2 and 3 ). 


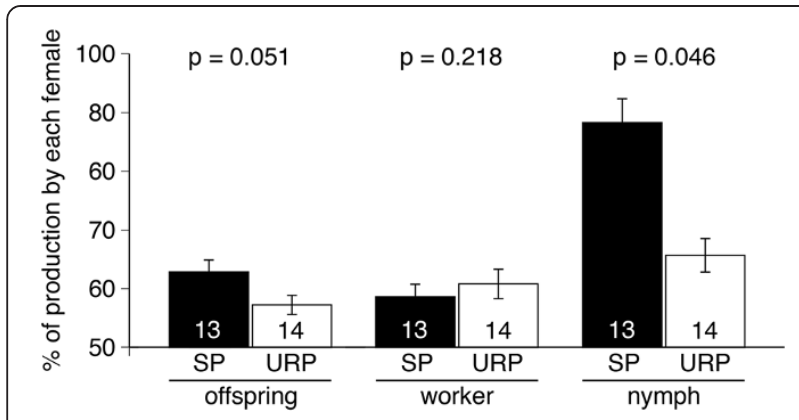

Figure 3 Proportions of offspring (including all individuals except for queens), workers, and nymphs produced by one of paired founding females. The proportions (means \pm S.D. values) were compared between sister-pair (SP, black) and unrelated-pair (URP, white) colonies using GLMM analyses. Numerical values in the bar indicate numbers of colonies examined.

According to the Red Queen Hypothesis for the evolution of sexual reproduction, genetic variation generated by sexual recombination among host individuals prevents pathogens from adapting to particular host genotypes [24-26]. In social insects intracolonial genetic variation among workers is thought to decrease intracolonial transmission of genotype-specific pathogens [27,28], and effective division of labor, particularly hygienic behavior such as corpse removal and colony cleaning, can prevent the spread of infectious diseases in insect colonies [29]. Intracolonial genetic variation is known to promote task specialization among workers [18,30-32], suggesting that genetic variation can improve disease resistance through division of labor. In social hymenopterans, worker genetic variation can be increased not only by sexual recombination but also by polyandry and/or polygyny [1]. In the basal termite Zootermopsis angusticollis, outbred colonies were more resistant to fungal and microbial pathogens than inbred colonies, indicating that genetic variation arising from outbreeding increased disease resistance through social interactions such as trophallaxis and grooming [33,34]. Because $R$. speratus at the colony founding stage faces high risk of infection by bacteria, fungi and natural pathogens [7], increased levels of genetic variation are expected to have an impact on survivorship during colony foundation. Thus, worker genetic variation stemming from pleometrosis between unrelated females may promote disease resistance.

\section{Reproductive skew in nymph production}

In $R$. speratus, the production of nymphs is a key step in colony reproduction, because nymphs can molt into alates or differentiate into nymphoid reproductives when primary reproductives senesce or die. Our results indicate that a skew in nymph production was positively correlated with relatedness of the individuals in female pairs (Figure 3); this supports one of the transactional models of reproductive skew (the concession model) in which a dominant individual controls the subordinate's fraction of total group reproduction [9]. According to the concession model, reproductive skew may be further increased under strong ecological constraints, when it would be preferable for the subordinate to stay in the colony rather than to leave the colony and reproduce alone [35]. It has been demonstrated that, in $R$. speratus, a female that founds a colony alone exhibits twice the mortality of a female in a founding pair, even under experimental conditions [6]. Although to date there have been few studies supporting these predictions of concession models [4], the current study strongly supports them. Skew models incorporate many parameters (e.g., relatedness, group size, fighting ability, ecological harshness on independent reproduction), of which total experimental control in order to fit to the assumptions is often less feasible. Our results supporting concession models might be due to our system, which more closely fits the models' assumptions (e.g. two cooperative individuals) than other systems. The current study additionally sheds light on a new aspect. There was a statistically significant colony growth $x$ relatedness interaction effect on reproductive skew (Table 2), suggesting that these are not independent and thus should be studied simultaneously. Future theoretical studies should focus on the possibility that if an enhanced colony's resource level is due to cooperation of unrelated females rather than related females, it can in turn increase the skew or vice versa (Additional file 4). Those are new facets of reproductive skew theory.

Parthenogenetically produced offspring are genetically predisposed to develop into nymphs in $R$. speratus [16]. However, in the presence of nymphoid reproductives, nymphal development is inhibited in one quarter of the offspring; they instead become workers [16]. In the present study, c.a. 80 percent of the offspring developed into workers in incipient female-female colonies (Figure 2b). This suggests that interaction with reproductive individuals may inhibit nymphal development. The present study further demonstrated that nymph production was influenced by the relatedness between founding females, whereas the production of workers was not (Figure 3, Additional file 4). These results suggest that skewed nymph production in these termites is controlled via nepotistic regulation of nymphal development by reproductives, in which some offspring parthenogenetically produced by a founding female are released from the induction of worker development. Although the proximate mechanism of this regulation remains unclear, physical contact and pheromonal regulation are possibilities. 


\section{Conclusions}

Our study clearly revealed that, in incipient colonies founded by two females of $R$. speratus, increased intracolonial genetic variation had two distinct impacts on social characteristics. First, it improved colony growth while maintaining the nymph/worker caste ratio, probably through effective division of labor and resistance to diseases. Second, it decreased reproductive skew via nepotistic regulation of nymphal development. To our knowledge, this is the first report testing and demonstrating these effects of intracolonial genetic variation simultaneously. Simultaneous testing such as this can serve to link colony characteristics in insect societies with the evolutionary significance of intracolonial genetic variation.

\section{Methods}

\section{Termite colonies}

Twelve mature termite colonies were collected in Toyama and Ishikawa Prefectures, Japan, in April 2008-2010 (colony no. A to L). Log sections housing the termites were brought back to the laboratory and maintained in plastic boxes in constant darkness.

\section{Female-female colony establishment}

After the emergence of alates (winged adults) from these colonies, the sexes of individuals were determined using the morphology of abdominal tergites [36]. Adults that had shed their wings were chosen randomly and femalefemale pairs were established (see [37]) with individuals from the same colony (using 4 colonies, total 28 sisterpairs) and from two different colonies (using 10 colonies, total 43 unrelated-pairs). Each pair was placed in a $20 \mathrm{~mL}$ glass vial with c.a. $8 \mathrm{~g}$ of mixed sawdust food (Mitani, Ibaraki, Japan) and kept at $25^{\circ} \mathrm{C}$ in constant darkness. At 15 months after colony foundation, 1802 and 3862 termites from 28 sister-pair and 43 unrelated-pair colonies, respectively, were sampled and stored in $99.5 \%$ ethanol. After determining their developmental stages and castes, 614 and 746 termites from the sister-pair and the unrelated-pair colonies were used for genotyping (see "Genotype analysis").

\section{DNA extraction and amplification}

Total DNA was extracted from the entire insect (larvae and eggs), a leg (queens) or the head (other castes), using the DNeasy Tissue Kit (Qiagen, Japan) for queens, and 10\% Chelex 100 (Bio-Rad, USA) for other individuals. Five microsatellite loci (Rf6-1, Rf21-1, Rf24-2, Rs02, and Rs03) were used for this study. Primer sequences for the amplification of Rf- and Rs-loci are given in Vargo [38] and Hayashi et al. [39], respectively.

\section{Genotype analysis}

First, genotypes of founding queens in each colony were analyzed using the five microsatellite loci. Three unrelatedpair colonies without surviving queens were excluded from this analysis. For 12 sister-pair colonies and 24 unrelatedpair colonies that contained only 1 surviving queen and offspring, we inferred the genotype of the dead queen from data of the existing queen and workers. If we could not obtain evidence that a dead queen produced eggs or larvae, we excluded these colonies from analysis; in such cases a queen had died at least 1 month prior to sampling. Based on the queens' genotyping data, 13 sister-pair colonies and 14 unrelated-pair colonies, in which genotypes of both founding queens were distinguished, were used for this analysis. Then, PCR products of 614 and 746 termites from the sister-pair and the unrelated-pair colonies were electrophoresed on $7 \%$ polyacrylamide gels ( $7 \mathrm{~h}, 100 \mathrm{~V}$ and $15 \mathrm{~mA}$ ), and stained with an ethidium bromide solution $(10 \mathrm{mg} / \mathrm{ml})$. Amplified DNA fragments were observed using Dolphin-Doc (Kurabo, Japan), and genotyping was performed with the use of Dolphin-1D software (Kurabo).

\section{Statistical analyses}

For evaluating how intracolonial genetic diversity affects social characteristics in $R$. speratus colonies, generalized linear mixed model (GLMM) analyses were conducted. Fixed effects were colony type (i.e., relatedness between founding queens), founders' survival rate, colony size, and their interactions, while founded colony and founders' source colony were included as random effects. Numbers of termites were analyzed using GLMMs with Poisson error distribution and log link function, whereas data for reproductive skew (number of offspring produced by more productive or less productive founders) and caste ratio were analyzed with binomial error distribution and logit link function. Significance of partial regression coefficients was evaluated by Wald tests. P values of less than 0.05 were considered significant. These analyses were conducted using $\mathrm{R}$ ver. 3.1.0 (available at http://cran.r-project. org/) and the lme4 package.

\section{Additional files}

Additional file 1: Inferred numbers of offspring produced by each female in sister-pair colonies.

Additional file 2: Inferred numbers of offspring produced by each female in unrelated-pair colonies.

Additional file 3: Proportions of juveniles (including eggs and larvae), soldiers, and nymphoids produced by one of paired founding females.

The proportions (means \pm S.D. values) were compared between sister-pair (SP, black) and unrelated-pair (URP, white) colonies using GLMM analyses. Numerical values in the bar indicate numbers of colonies examined.

Additional file 4: Skew of nymph production affected by the interactions among colony type, colony size and founders' survival rate. 
In unrelated-pair colonies, colony size increased the skew of nymph production, regardless of the number of founding females (narrow solid and broken lines). In sister-pair colonies with both founding females, colony size increased the skew (thick solid line). In sister-pair colonies where only one of two founding females survived, colony size decreased the skewed nymph production (thick broken line). Here, the interactions between colony type and colony size, and also interactions among these two and founders' survival rate significantly affected the skew of nymph production (see Table 2).

\section{Competing interests}

The authors declare that they have no competing interests.

\section{Authors' contributions}

SM and MY performed genetic analyses and drafted the manuscript. RS carried out the ecological experiments and prepared Figures 1,2 and 3. YH and OK analyzed molecular data. KT participated in the design of the study and performed the statistical analysis. KM conceived of the study, and participated in its design and coordination and helped to draft the manuscript. All authors read and approved the final manuscript.

\section{Acknowledgements}

We are grateful to Dr. Christine Nalepa who corrected English and gave us valuable comments. We also thank all laboratory members for their help in our sampling and their assistance in laboratory work. This study was supported in part by Grant-in-Aids for Scientific Research (C) (No. 24570022 to KM) from the Japan Society for the Promotion of Science.

\section{Author details}

'Graduate School of Science and Engineering, University of Toyama, 930-8555 Gofuku, Toyama, Japan. ${ }^{2}$ Department of Hygiene and Public Health, Tokyo Women's Medical University, 162-8666 Shinjuku-ku, Tokyo, Japan. ${ }^{3}$ Graduate School of Environmental Science, Hokkaido University, 060-0810 Sapporo, Hokkaido, Japan. ${ }^{4}$ College of Science, Ibaraki University, 310-8512 Mito, Ibaraki, Japan. ${ }^{5}$ Faculty of Agriculture, University of the Ryukyus, 903-0213 Nishihara, Okinawa, Japan.

Received: 25 July 2014 Accepted: 29 July 2014

Published: 14 August 2014

\section{References}

1. Oldroyd BP, Fewell JH: Genetic diversity promotes homeostasis in insect colonies. Trends Ecol Evol 2007, 22(8):408-413.

2. King KC, Lively CM: Does genetic diversity limit disease spread in natura host populations? Heredity 2012, 109(4):199-203.

3. Schmid-Hempel P: Infection and colony variability in social insects. Philos Trans R Soc B 1994, 346(1317):313-321.

4. Nonacs $P$, Hager R: The past, present and future of reproductive skew theory and experiments. Biol Rev 2011, 86(2):271-298.

5. Nutting WL: Flight and colony foundation. In Biology of Termites. Volume 1. Edited by Krishna K, Weesner FM. New York: Academic; 1969:223-282.

6. Matsuura K, Nishida T: Comparison of colony foundation success between sexual pairs and female asexual units in the termite Reticulitermes speratus (Isoptera: Rhinotermitidae). Popul Ecol 2001, 43(2):119-124

7. Matsuura K, Fujimoto M, Goka K, Nishida T: Cooperative colony foundation by termite female pairs: altruism for survivorship in incipient colonies. Anim Behav 2002, 64(2):167-173.

8. Matsuura K, Fujimoto M, Goka K: Sexual and asexual colony foundation and the mechanism of facultative parthenogenesis in the termite Reticulitermes speratus (Isoptera, Rhinotermitidae). Insectes Soc 2004, 51(4):325-332.

9. Reeve HK, Keller L: Tests of reproductive-skew models in social insects. Annu Rev Entomol 2001, 46(1):347-385.

10. Nonacs P: Kinship, greenbeards, and runaway social selection in the evolution of social insect cooperation. Proc Natl Acad Sci 2011 108(Supplement 2):10808-10815.

11. Reeve HK, Emlen ST, Keller L: Reproductive sharing in animal societies: reproductive incentives or incomplete control by dominant breeders? Behav Ecol 1998, 9(3):267-278.

12. Kokko H: Review paper: are reproductive skew models evolutionarily stable? Proc Biol Sci 2003, 270(1512):265-270.
13. Johnstone RA: Models of reproductive skew: a review and synthesis (invited article). Ethology 2000, 106(1):5-26.

14. Tsuji K, Kasuya E: What do the indices of reproductive skew measure? Am Nat 2001, 158(2):155-165.

15. Reeve H, Ratnieks F: Queen-queen conflict in polygynous societies: mutual tolerance and reproductive skew. In Queen Number and Sociality in Insects. Edited by Keller L. Oxford, UK: Oxford University Press; 1993:45-85.

16. Hayashi Y, Lo N, Miyata H, Kitade O: Sex-linked genetic influence on caste determination in a termite. Science 2007, 318(5852):985-987.

17. Matsuura K, Vargo EL, Kawatsu K, Labadie PE, Nakano H, Yashiro T, Tsuji K: Queen succession through asexual reproduction in termites. Science 2009, 323(5922):1687.

18. Julian $\mathrm{GE}$, Fewell JH: Genetic variation and task specialization in the desert leaf-cutter ant, Acromyrmex versicolor. Anim Behav 2004, 68(1):1-8.

19. Cole BJ, Wiernasz DC: The selective advantage of low relatedness. Science 1999, 285(5429):891-893.

20. Wiernasz DC, Hines J, Parker DG, Cole BJ: Mating for variety increases foraging activity in the harvester ant, Pogonomyrmex occidentalis. Mol Ecol 2008, 17(4):1137-1144.

21. Goodisman MAD, Kovacs JL, Hoffman EA: The significance of multiple mating in the social wasp Vespula maculifrons. Evolution 2007, 61(9):2260-2267.

22. Moreau CS, Bell CD, Vila R, Archibald SB, Pierce NE: Phylogeny of the ants: diversification in the age of angiosperms. Science 2006, 312(5770):101-104.

23. Hines HM, Hunt JH, O'Connor TK, Gillespie JJ, Cameron SA: Multigene phylogeny reveals eusociality evolved twice in vespid wasps. Proc Natl Acad Sci 2007, 104(9):3295-3299.

24. van Valen L: A new evolutionary law. Evol Theory 1973, 1:1-30.

25. Ebert D, Hamilton WD: Sex against virulence: the coevolution of parasitic diseases. Trends Ecol Evol 1996, 11(2):79-82.

26. Smith JM: What use is sex? J Theor Biol 1971, 30(2):319-335.

27. Shykoff J, Schmid-Hempel P: Genetic relatedness and eusociality: parasitemediated selection on the genetic composition of groups. Behav Ecol Sociobiol 1991, 28(5):371-376

28. Shykoff JA, Schmid-Hempel P: Parasites and the advantage of genetic variability within social insect colonies. Proc Biol Sci 1991, 243(1306):55-58

29. Schmid-Hempel P: Parasites in Social Insects. Princeton, NJ: Princeton University Press; 1998.

30. Hughes WO, Sumner S, Van Borm S, Boomsma JJ: Worker caste polymorphism has a genetic basis in Acromyrmex leaf-cutting ants. Proc Natl Acad Sci 2003, 100(16):9394-9397.

31. Rheindt FE, Strehl CP, Gadau J: A genetic component in the determination of worker polymorphism in the Florida harvester ant Pogonomyrmex badius. Insectes Soc 2005, 52(2):163-168.

32. Robinson GE, Page RE: Genetic determination of guarding and undertaking in honey-bee colonies. Nature 1988, 333(6171):356-358

33. Calleri DV, McGrail Reid E, Rosengaus RB, Vargo EL, Traniello JFA: Inbreeding and disease resistance in a social insect: effects of heterozygosity on immunocompetence in the termite Zootermopsis angusticollis. Proc Biol Sci 2006, 273(1601):2633-2640.

34. Traniello JFA, Rosengaus RB, Savoie K: The development of immunity in a social insect: evidence for the group facilitation of disease resistance. Proc Natl Acad Sci 2002, 99(10):6838-6842.

35. Reeve HK, Ratnieks FLW: Queen-Queen Conflicts in Polygynous Societies: Mutual Tolerance and Reproductive Skew. Oxford, UK: Oxford University Press; 1993.

36. Weesner FM: The reproductive system. In Biology of Termites. Volume 1. Edited by Krishna K, Weesner FM. New York: Academic; 1969:125-160.

37. Ishitani K Maekawa K: Ovarian development of female-female pairs in the termite, Reticulitermes speratus. J Insect Sci 2010, 10(194):1-12.

38. Vargo EL: Polymorphism at trinucleotide microsatellite loci in the subterranean termite Reticulitermes flavipes. Mol Ecol 2000, 9(6):817-820.

39. Hayashi Y, Kitade O, Kojima J-I: Microsatellite loci in the Japanese subterranean termite, Reticulitermes speratus. Mol Ecol Notes 2002, 2(4):518-520.

doi:10.1186/s12862-014-0177-0

Cite this article as: Miyazaki et al.: Intracolonial genetic variation affects reproductive skew and colony productivity during colony foundation in a parthenogenetic termite. BMC Evolutionary Biology 2014 14:177. 